\title{
Perspectives on current uses of the AHRQ quality indicators and future development
}

The Agency for Healthcare Research and Quality (AHRQ) is the lead United States Federal agency charged with improving the quality, safety, efficiency, and effectiveness of health care for all Americans. The AHRQ supports health services research that will improve the quality of health care and promote evidence-based decision-making.

The AHRQ Quality Indicators (AHRQ QIs), which are based on administrative data, were initially designed for quality tracking and improvement and are extensively used for these purposes. While the focus of initial measure development was not on hospital-level comparative measurement, the increased demand for such metrics in a time of growing quality concerns has led to their adaptation and adoption for these purposes. Currently, the three primary uses of the AHRQ QIs are for the following:

1. Quality assessment and improvement;

2. Public Reporting; and

3. Quality based purchasing (commonly known as „pay for performance“ in the United States)

Use of the AHRQ QIs for quality assessment and improvement is being applied by various entities at a variety of levels of measurement. For example, States use the measures to understand performance in a number of clinical areas in aggregate on the State level as well as at a more granular level, such as the county. A number of State hospital organizations and health care systems measure the AHRQ QIs at the hospital level and report the results back to their affiliated hospitals for their use in quality monitoring and improvement.

Public reporting at the hospital level has flourished in recent years. The AHRQ QIs play a prominent role in a number of public reports. Through public reports of hospital level performance in 16 states currently, over half of the United States population has access to quality information on hospitals in their state.

Increasingly purchasers of health care (private purchasers, health plans, Medicare) are linking a portion of the payment to quality. Payment is used to recognize high performance, such as reaching a set standard in a risk adjusted complication rate. More recently payment is also being used to reward improvement, such as a hospital experiencing a lower risk adjusted mortality rate from the prior year to the current year. The AHRQ QIs are being used by a number of organizations in their quality based purchasing system.

There is a continuous effort to revise, update and improve the AHRQ QIs as the science of measurement and the data evolve. A recent example is in regard to the availability of present on admission (POA) indications for all diagnosis codes in the administrative record. The addition of POA indications has great potential to increase the precision of the measurement of adverse outcomes of care. The ARHQ QIs presently include a methodology to incorporate POA indications.

In addition to ongoing refinement efforts of the measures is the expansion and adaptation of the existing measure sets. At the present time there are four AHRQ QI modules: 1) Inpatient Quality Indicators (IQIs), 2) Patient Safety Indicators (PSIs), 3) Pediatric Quality Indicators (PDIs), and 4) Prevention Quality Indictors (PQIs). Future work include the addition of the following AHRQ QI modules: 1) efficiency measures, 2) care coordination measures, 3) Emergency Department PSIs, 4) Emergency Department PQIs, 5) PQIs for the Medicaid Home and Community Based Services program, and 6) Health Plan PQIs.

Author's declaration: The author does not have any financial interests or conflicts of interest relevant to the subject of this manuscript.
J. Bott

Qualitätsmanagement

Keywords

quality

Oindicators

measurement

Schlüsselwörter

Qualität

Ondikatoren

Qualitätsmessung 